\title{
Phylogeny, sequence-typing and virulence profile of uropathogenic Escherichia coli (UPEC) strains from Pakistan
}

\author{
Ihsan Ali5', Zara Rafaque ${ }^{1 \dagger}$, Ibrar Ahmed ${ }^{2}$, Faiza Tariq ${ }^{1}$, Sarah E. Graham³ ${ }^{3}$ Elizabeth Salzman ${ }^{4}$, Betsy Foxman ${ }^{4}$ and
} Javid lqbal Dasti ${ }^{1 *}$

\begin{abstract}
Background: Escherichia coli lineage ST131 predominates across various spectra of extra-intestinal infections, including urinary tract infection (UTI). The distinctive resistance profile, diverse armamentarium of virulence factors and rapid global dissemination of ST131 E. coli makes it an intriguing pathogen. However, not much is known about the prevalence and genetic attributes of ST131 lineage in Pakistan.

Methods: We estimated prevalence and genetic attributes of E. coli ST131 isolates causing UTI among 155 randomly selected samples. Samples were analyzed for phylogenetic grouping, O-typing and fumC/fimH typing. Isolates were further tested for the ESBL and virulence factors using PCR.

Results: Overall, $59 \%$ of the UPEC isolates belonged to the phylogenetic group B2, followed by D $=28 \%, B 1=8 \%$ and $A=5 \%$. Among 18 different Sequence-types, ST131 was the dominant lineage $(n=71 ; 46 \%)$ out of which $72 \%$ of the isolates were assigned to the phylogenetic group B2, while $61 \%$ adhered to the serogroup O25b. FumC/fimH typing confirmed 49\% of the ST131 as H30 sub-types. In this study, significant numbers of the identified ST131 isolates were MDR and $42 \%$ showed ESBL phenotypes, out of which 37\% carried bla- $\mathrm{CTX-M-15}$. Moreover, different virulence factors were detected in following percentages: fimH,155(100\%), iutA 86 (55\%), feoB 76 (49\%), papC 75 (48\%), papGll 70 (45\%), kpsMTIl 40 (26\%), papEF 37 (24\%), fyuA 37 (24\%), usp 22 (14\%), papA 20 (13\%), sfa/foc20 (13\%), hlyA 18 (12\%), afa 15 (10\%), cdtB 11 (7\%), papGl 6 (4\%), papGIII 6 (4\%), kpsMTIII 4 (3\%) and bmaE2 (1\%).

Conclusion: Conclusively, this study provides important insight into the genetic and virulence attributes of pandemic MDR ST131 strains involved in UTIs. It also highlights higher prevalence of ST131-O25b-H30 UPEC isolates in patients, which was previously unreported from this part of globe.
\end{abstract}

Keywords: ST131, VF genes, ESBL, UPEC, MDR

\section{Background}

Extra-intestinal E. coli is the major cause of urinary tract infections and resistance among UTI strains has been mounting against different antibiotics, including trimethoprimsulfamethoxazole, fluoroquinolones extended spectrum cephalosporins and amoxicillin clavulanic acid [1-3]. Due to the emergence of specific clonal groups such as ST131, global dissemination of fluoroquinolone-resistance was

\footnotetext{
* Correspondence: iqbal78@hushmail.com

${ }^{+}$Ihsan Ali and Zara Rafaque contributed equally to this work.

'Department of Microbiology, Faculty of Biological Sciences, Quaid-i-Azam

University, Islamabad 45320, Pakistan

Full list of author information is available at the end of the article
}

highlighted across different geographical regions [4-6]. Clonal group ST131 predominates across various spectra of infections including cystitis, pyelonephritis, bacteremia, meningitis, septic shock, epididymo-orchitis and osteoarticular infection. [7, 8]. In addition, ST131 strains harbor diverse armamentarium of virulence factors and their genetic homogeneity regarding virulence potential and resistance profile has been widely endorsed [8]. Notably, a subgroup of ST131 strains, known as H30-Rx has remarkable tendency to encode bla- ${ }_{C T X-M-15}$ gene $[7,9,10]$. In the current scenario of global urgency related to the antibiotic resistance, underlying epidemiological factors related to the fitness and fast emergence of ST131 across different regions

(c) The Author(s). 2019 Open Access This article is distributed under the terms of the Creative Commons Attribution 4.0 International License (http://creativecommons.org/licenses/by/4.0/), which permits unrestricted use, distribution, and reproduction in any medium, provided you give appropriate credit to the original author(s) and the source, provide a link to the Creative Commons license, and indicate if changes were made. The Creative Commons Public Domain Dedication waiver (http://creativecommons.org/publicdomain/zero/1.0/) applies to the data made available in this article, unless otherwise stated. 
are under intensive scrutiny. However, in Pakistan phylogenetic grouping, sequence types, virulence attributes and antibiotic susceptibility profile of UPEC strains remains unexplored [11, 12]. Therefore, data related to the clonal types and resistance profile of the strains involved in urinary tract infections in Pakistan is extremely scarce. This study fills the gap and provides important insights into the genetic and virulence attributes of pandemic MDR ST131 strains involved in UTIs in Pakistan.

\section{Methods}

\section{Sample collection and antibiotic susceptibility testing}

Altogether $n=155$ identified uropathogenic E. coli (UPEC) were collected during the period of August 2012 to August 2014, from Pakistan Institute of Medical Sciences. Ethical Review Board (ERB) of Pakistan Institute of Medical Sciences approved this study. Ethical Review Board approved verbal consent taken from all the patients. Important patient data such as name, age, gender, location was recorded and unique identification number were assigned to each patient. Samples were from community-acquired urinary tract infections. Antibiotic testing and phenotypic detections of ESBL were performed by disc diffusion methods according to the guidelines CLSI, 2014 [13]. Isolates were tested for the susceptibility to 12 different classes of antibiotics including $\beta$-lactamase inhibitors (piperacillin tazobactam, amoxicillin-clavulanic acid), cephalosporins (ceftazidime, cefotaxime, ceftriaxone), fluoroquinolone (ciprofloxacin, levofloxacin), aminoglycosides (amikacin), trimethoprim sulfonamides, nitrofurantoin, and fosfomycin (BIOANALYSE, Turkey). Control strain E. coli ATCC 25922 was used in this assay.

\section{Phylogeny, serotyping, and fumC/fimH typing}

We used the procedure reported by Clermont et al, 2000 to perform phylogenetic analysis of 155 isolates [14]. FumC/fimHtyping ( $\mathrm{CH}$ typing) was performed as previously described [15]. Briefly, PCR amplifications were carried out in $25 \mu \mathrm{l}(12.5 \mu \mathrm{l}$ GoTaq DNA polymerase (Promega), $7.5 \mu \mathrm{l}$ water, $1 \mu \mathrm{l}$ bacterial DNA, $2 \mu \mathrm{l}$ of each forward and reverse primers). The amplified products were analyzed on $2 \%$ agarose gel. The confirmed PCR products were purified using PCR purification kit (QIAquick, QIAGEN) and all the amplified DNA fragments were sequenced (ABI 3130, Perkin-Elmer Applied Biosystems, Foster City, California). The forward and reverse sequences were aligned, trimmed off using Codon Code Aligner and results were compiled according to the standard procedures $[15,16]$. Additionally, by targeting $347 \mathrm{bp}$ of pabB gene fragment, clonal group ST131 was scrutinized for serogroup O25b [17]. Previously typed O25b-ST131 strains and K-12 E. coli were included as experimental controls in this study.

\section{Detection of $\beta$-lactamases and virulence factor genes}

In order to detect extra-chromosomally encoded ESBL factors, plasmid DNA was isolated by commercially available kit (Thermo-Scientific Gene Jet plasmid Miniprep Kit). ESBL factors including $b l a_{\text {-TEM }}, b l a_{\text {-SHV }}$ and genes $b l a_{\text {-OXA }}$, bla $_{\text {-PSE }}$ were PCR amplified as described elsewhere [18]. Amplified products were then purified (Gel Band Purification Kit, Amersham, USA) and sequencing was done by automated DNA sequencer (ABI 3130, Perkin-Elmer Applied Biosystems, Foster City, California). Sequences were reported to the Gene Bank database (accession number; KX171170-171195). PCR amplifications and sequencing of bla-СтХ-м allele was carried out, $b l a_{- \text {СтХ-м }}$ types were determined by comparing DNA sequences available in the database [19]. A total of 18 different virulence factors (VF) corresponding to the main classes of extra-intestinal virulence associated genes (VAGs) including adhesins, toxins, siderophores, capsular proteins and uropathogenic-specific protein (usp) were scrutinized in all 155 isolates. VF genes were amplified by previously reported sets of primers and amplification conditions [20].

\section{Statistical analysis}

The statistical analysis was performed using Graph Pad Prism, version 7. Both Chi square and Fisher exact tests were used to assess differences by assuming cut-off value of $P<0.05$ as significant.

\section{Results}

\section{Phylogeny and sequence typing}

Overall, phylogenetic group B2 showed highest representation, 92(59\%) followed by D 43 (28\%), B1 12 (8\%) and A $8(5 \%)$ (Table 1). Eighteen different sequence types (STs) of 152 isolates were confirmed, constituting $98 \%$ of all the isolates; the remaining $2 \%$ of the isolates were un-typeable. Clonal group ST131 comparised 71(46\%) of all the isolates, followed by two other lineages, ST405 28(18\%) and ST168 $16(10 \%)$ (Table 2). Majority of the ST131 strains $51(72 \%)$ belonged to the phylogenetic group B2, while $43(61 \%)$ were assigned to serogroup O25b. CH typing confirmed 35(49\%) as ST131-H30 sub-group of strains, out of which 22(31\%) belonged to the serogroup O25b.

\section{MDR among ST131 strains}

pt? $>$ Significant number of the isolates assigned to the phylogenetic group B2 and D were multi-drug resistant (Table 2). Similarly, among different STs including ST131, ST405, ST168, ST29, ST69 and ST89, significant number of the isolates were multi-drug resistant (Table 2). The tendency of ESBL production and the fluoroquinolone resistance was relatively higher among ST131 isolates and majority of these isolates were multi-drug resistant (Table 2). Resistance against nitrofurantoin was significantly higher among ST131 isolates in comparison to the other sequence types, whereas one of 
Table 1 Distribution of ESBL factors, antibiotic resistance and VF genes in MDR UPEC

\begin{tabular}{|c|c|c|c|c|c|c|}
\hline \multicolumn{7}{|c|}{ Numbers and percentagesof isolates and their respective traits $(n=155)$} \\
\hline Resistance traits & Total Isolates $(n=155) n(\%)$ & $\begin{array}{l}\text { Group A } \\
(n=8) \mathrm{n}(\%)\end{array}$ & $\begin{array}{l}\text { Group B1 } \\
(n=12) n(\%)\end{array}$ & $\begin{array}{l}\text { Group B2 } \\
(n=92) n(\%)\end{array}$ & $\begin{array}{l}\text { Group D } \\
(n=43) n(\%)\end{array}$ & $p$ value \\
\hline ESBL phenotypes & $65(42)$ & $1(13)$ & $3(25)$ & $36(39)$ & 25(58) & 0.0268 \\
\hline blactX-M-15 & $57(37)$ & $1(13)$ & $3(25)$ & $32(35)$ & $21(49)$ & 0.1333 \\
\hline blaTEM & 23(15) & $1(13)$ & $3(25)$ & $13(14)$ & $6(14)$ & 0.7823 \\
\hline blaSHV & $6(4)$ & 00 & 00 & 3(3) & $3(7)$ & 0.0907 \\
\hline blaOXA & $10(6)$ & 00 & $1(8)$ & $4(4)$ & $5(12)$ & 0.3610 \\
\hline blaPSE & $1(0.6)$ & 00 & 00 & $1(1)$ & 00 & 0.8783 \\
\hline Piperacillin tazobactam & $7(5)$ & 00 & $1(8)$ & $4(4)$ & $2(5)$ & 0.8514 \\
\hline Ceftazidime & $96(62)$ & $3(38)$ & $7(58)$ & $54(59)$ & $32(74)$ & 0.1483 \\
\hline Cefotaxime & $101(65)$ & $3(38)$ & $7(58)$ & $59(64)$ & $32(74)$ & 0.2028 \\
\hline Ceftriaxone & $99(64)$ & $3(38)$ & $8(67)$ & $59(64)$ & $29(67)$ & 0.4001 \\
\hline Ciprofloxacin & $95(61)$ & $6(75)$ & $7(58)$ & $56(90)$ & $26(60)$ & 0.4001 \\
\hline Levofloxacin & $97(63)$ & $7(88)$ & $8(67)$ & $56(90)$ & $26(60)$ & 0.4929 \\
\hline Amikacin & $7(5)$ & $1(13)$ & 00 & $5(5)$ & $1(2)$ & 0.4920 \\
\hline Gentamicin & $47(30)$ & $4(50)$ & $6(50)$ & $24(26)$ & 13(30) & 0.2171 \\
\hline Amoxicillin-clavulanic acid & $111(72)$ & $5(63)$ & $7(58)$ & $66(72)$ & $26(60)$ & 0.5224 \\
\hline Trimethoprim sulfonamides & 130(84) & $7(88)$ & $11(92)$ & $77(84)$ & $35(81)$ & 0.8461 \\
\hline Nitrofurantoin & $9(6)$ & $1(13)$ & $1(8)$ & $6(7)$ & $1(2)$ & 0.6075 \\
\hline Fosfomycin & $15(10)$ & $1(13)$ & $2(17)$ & $8(9)$ & $4(9)$ & 0.8370 \\
\hline $\mathrm{fimH}$ & $155(100)$ & $8(100)$ & $12(100)$ & $92(100)$ & $43(100)$ & $>0.9999$ \\
\hline papA & $20(13)$ & $1(13)$ & $1(8)$ & $12(13)$ & $6(14)$ & 0.9659 \\
\hline papC & $75(48)$ & $2(25)$ & $8(67)$ & $43(47)$ & $22(51)$ & 0.3092 \\
\hline papEF & $37(24)$ & 0 & $6(50)$ & $19(21)$ & $12(28)$ & 0.0476 \\
\hline papGl & $6(4)$ & 0 & 0 & $3(3)$ & $3(7)$ & 0.5699 \\
\hline papGll & $70(45)$ & $2(25)$ & $7(58)$ & $37(40)$ & $24(53)$ & 0.1695 \\
\hline papGIII & $6(4)$ & 0 & 0 & $4(4)$ & $2(5)$ & 0.8177 \\
\hline sfa/foc & $20(13)$ & $2(25)$ & $1(8)$ & $13(14)$ & $4(9)$ & 0.5968 \\
\hline Afa & $15(10)$ & 0 & $1(8)$ & $10(11)$ & $4(9)$ & 0.7919 \\
\hline bmaE & $2(1)$ & 0 & $1(8)$ & $1(1)$ & 0 & 0.1466 \\
\hline fyuA & $37(24)$ & $1(13)$ & $2(17)$ & $21(23)$ & $13(30)$ & 0.5882 \\
\hline iutA & $86(55)$ & $5(63)$ & $5(42)$ & $52(57)$ & $24(56)$ & 0.7701 \\
\hline$f e o B$ & $76(49)$ & $3(38)$ & $6(50)$ & $40(43)$ & $27(63)$ & 0.1852 \\
\hline kpsmtll & $40(26)$ & 0 & $4(33)$ & $21(23)$ & $15(35)$ & 0.1438 \\
\hline kpsmtlll & $4(3)$ & 0 & $1(8)$ & $3(3)$ & 0 & 0.3765 \\
\hline Usp & $22(14)$ & $2(25)$ & $2(17)$ & $14(15)$ & $4(9)$ & 0.6256 \\
\hline hlyA & $18(12)$ & $1(13)$ & $3(25)$ & $6(7)$ & 8 (19) & 0.0908 \\
\hline$c d t B$ & $11(7)$ & 0 & $3(25)$ & $6(7)$ & $2(5)$ & 0.0758 \\
\hline
\end{tabular}

Distribution of resistance and virulence traits among different phylogroups of uropathogenic $E$. coli $(N=155)$. The $p$ values were calculated by comparing different traits among phylogroups

the frequently prevalent sequence types, ST168 strains were significantly resistant to levofloxacin (Table 3 ). Resistance against carbapenemes has not been evaluated for the scrutinized strains in this study; hence it is beyond the scope of this discussion.

\section{Occurrence of $\beta$-lactamases among ST131}

Overall, occurrence of ESBL was higher among clonal group ST131, constituting $42 \%$ of the total ESBL phenotypes (Table 3). Moreover, $78 \%$ of the ESBL phenotypes showed resistance to at least one 
Table 2 Distribution of MDR and fluoroquinolone resistant MDR strains in different phylogroups and ST-types

\begin{tabular}{|c|c|c|c|}
\hline \multicolumn{4}{|c|}{ Numbers and percentages of the sequence typed isolates $(n=155)$} \\
\hline $\begin{array}{l}\text { No of isolates in } \\
\text { group } n(\%)\end{array}$ & $\begin{array}{l}\text { No of MDR } \\
\text { isolates n (\%) }\end{array}$ & $\begin{array}{l}\text { ESBL+FQR-MDR } \\
\text { isolates } n(\%)\end{array}$ & $p$ value \\
\hline Group A 8(5) & $7(88)$ & $2(25)$ & 0.0117 \\
\hline Group B112(8) & 10(83) & $5(42)$ & 0.0350 \\
\hline Group B2 92(59) & $71(77)$ & $33(36)$ & $<0.0001$ \\
\hline Group D 43(28) & $36(84)$ & $11(26)$ & $<0.0001$ \\
\hline ST131 71(46) & $57(82)$ & $22(31)$ & $<0.0001$ \\
\hline H30 35(49) & $28(80)$ & 10(29) & $<0.0001$ \\
\hline Non H30 36(51) & 29(81) & 12(33) & $<0.0001$ \\
\hline ST405 28(18) & 24(86) & 10(36) & 0.0001 \\
\hline ST168 16(10) & 14(88) & $7(44)$ & 0.0092 \\
\hline ST2913(8) & $9(69)$ & $5(38)$ & 0.1156 \\
\hline ST69 5(3) & $5(100)$ & $2(40)$ & 0.0384 \\
\hline ST95 2(1) & $2(100)$ & 00 & \\
\hline ST31 2(1) & 00 & 00 & \\
\hline ST10 2(1) & $1(50)$ & 00 & \\
\hline ST4482(1) & $1(50)$ & 00 & \\
\hline ST892(1) & $2(100)$ & $1(50)$ & 0.2482 \\
\hline ST7032(1) & $2(100)$ & 00 & \\
\hline ST910 1(1) & $1(100)$ & 00 & \\
\hline ST5451(1) & 00 & 00 & \\
\hline ST9711(1) & 00 & 00 & \\
\hline ST1531(1) & 00 & 00 & \\
\hline ST1521(1) & $1(100)$ & 00 & \\
\hline ST121(1) & $1(100)$ & 00 & \\
\hline ST8381(1) & $0(100)$ & 00 & \\
\hline NSC3(2) & $2(67)$ & $2(67)$ & $>0.9999$ \\
\hline
\end{tabular}

Phylogenetic and sequence type distribution of co-resistance among uropathogenic $E$. coli $(N=155)$. The $p$ values were calculated by comparing total number of MDR producers and ESBL producers FQR MDR

fluoroquinolone and 95\% were resistant to at least one cephalosporin. The occurrence of ESBL genes remained as follows, bla-CTX-M-15, 57(39\%), bla-TЕM 23(15\%) and bla-SHV 6(4\%). Prevalence of other $\beta$ lactamases genes such as $b a_{\text {-OXA }}$ and $b l a_{\text {-PSE }}{ }_{1}$ remained 6 and $0.6 \%$ respectively. In comparison to other sequence types, overall prevalence of $\beta$-lactamases genes was higher among ST131 strains, $27(38 \%)$ of the bla-CTX-M-15, followed by bla-TEM $8(11 \%)$, bla $a_{-\mathrm{SHV}} 3(4 \%)$ and bla-OXA $6(8 \%)$. Presence of bla-CTX-M-15 was highest (100\%) among ST131 H30-O25b and 91\% of the bla-CTX-M-15 positive ST131 H30-O25b isolates were resistant to fluoroquinolones (Data not shown). ESBL producing isolates were frequently found resistant to ceftazidime, cefotaxime, ceftriaxone, ciprofloxacin, levofloxacin, amoxicillinclavulanic acid and trimethoprim sulfonamides (Table 4).
Distribution of VF genes among different sequence types A total of 18 different virulence factors were scrutinized among 155 isolates. Percntages of VF genes were as follows: fim H,155(100\%), iutA86 (55\%), feo B 76 (49\%), papC 75 (48\%), papGII70 (45\%), kpsMTII 40 (26\%), papEF 37 (24\%), fyuA 37 (24\%), usp 22 (14\%), papA 20 (13\%), sfa/foc 20 (13\%), hlyA 18 (12\%), afa 15 (10\%), cdtB 11 (7\%), papGI 6 (4\%), papGIII 6 (4\%), kpsMTIII 4 (3\%) and bmaE2 (1\%). Some virulence factors such as $s f a / f o c$, fyuA and $f e o \mathrm{~B}$ were detected frequently among ST131 isolates whereas VF-genes papEF, sfa/focand hlyA were frequently associated with $H 30$ sub-clone (Table 5). Overall, virulence genes such as $\mathrm{sfa} / f o c, f y u \mathrm{~A}$ and $f e o \mathrm{~B}$ were associated significantly $(p<0.05)$ with ST131 strains, while papEF had significant presence among clonal group ST131-H30.

\section{Discussion}

E. coli ST131 was reported from three different continents [8]. However, recently it has become the most predominant lineage associated with variety of infections around the globe. ST131 strains have a tendency to harbor ESBL enzymes bla- ${ }_{\mathrm{CTX}-\mathrm{M}-15}$, which play a significant role in mounting resistance against $\beta$-lactam class of antibiotics [8]. Moreover, ST131 strains show remarkable resistance to the fluoroquinolones and demonstrate greater abilities to adhere bladder, kidneys and epithelial cells $[4,5]$. In this study, clonal group ST131 was the most prevalent lineage, comprising $46 \%$ of the isolates and majority of these isolates (59\%) belonged to the phylogenetic group B2. Prevalence of two other lineages ST405 and ST168 were 18 and $10 \%$ respectively. Involvement of these lineages in UTIs has been described earlier and recently ST405 was confirmed as an emerging uropathogenic E. coli clone in Saudi Arabia $[21,22]$. Urinary tract infections caused by $E$. coli pose considerable challenge and are associated with higher morbidity and mortality [5]. Due to their resistance against variety of antibiotics, including $\beta$-lactams, aminoglycosides and fluoroquinolones, infections caused by pandemic clonal group ST131 are particularly challenging to treat $[23,24]$. In this context, epidemiological significance of a sub group ST131 H30-Rx has been well described $[5,25]$. In this study, $50 \%$ of the ST131 strains carried $H 30$ variant of $f i m \mathrm{H}$ gene and $61 \%$ belonged to the serogroup O25b. All the isolates belonging to the sub-group ST131-H30-O25b carried ESBL blaCTX-M-15 however, overall prevalence of these particular strains constituted only $10 \%$ of the total isolates. Resistance against fluoroquinolones in ST131 strains remained $60 \%$, that was remarkably higher. For the treatment of UTIs commonly prescribed antibiotics include sulphamethoxazole-trimethoprim and fluoroquinolones. 


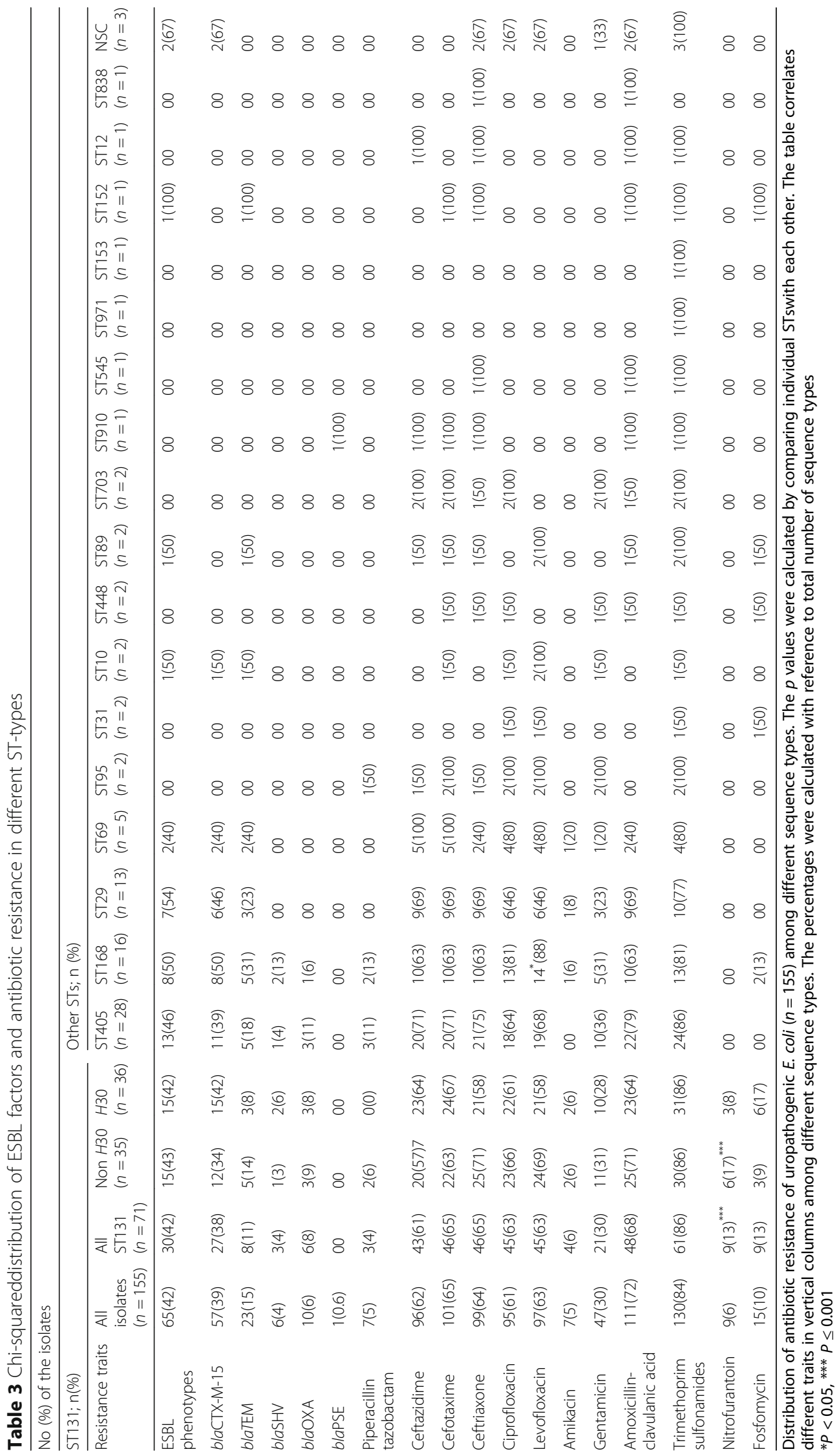


Table 4 Distribution of drug resistance and VF genes among ESBL producers and non ESBLUPEC

\begin{tabular}{|c|c|c|c|c|}
\hline \multicolumn{5}{|c|}{ Numbers and percentages of the isolates andtheir respective traits } \\
\hline Resistance traits & All isolates $(n=155) ; \mathrm{n}(\%)$ & Non ESBL producers $(n=90) \mathrm{n}(\%)$ & ESBL producers $(n=65) \mathrm{n}(\%)$ & $p$ value \\
\hline Piperacillin tazobactam & $7(5)$ & $4(4)$ & $3(5)$ & $>0.9999$ \\
\hline Ceftazidime & $96(62)$ & $36(40)$ & $60(92)$ & $<0.0001$ \\
\hline Cefotaxime & $101(65)$ & $40(44)$ & $61(94)$ & $<0.0001$ \\
\hline Ceftriaxone & $99(64)$ & $49(54)$ & $50(77)$ & 0.0040 \\
\hline Ciprofloxacin & $95(61)$ & $45(50)$ & $50(77)$ & 0.000684 \\
\hline Levofloxacin & $97(63)$ & $48(53)$ & $49(75)$ & 0.0051 \\
\hline Amikacin & $7(5)$ & $4(4)$ & $3(5)$ & 0.9597 \\
\hline Gentamicin & $47(30)$ & $30(33)$ & $17(26)$ & 0.3373 \\
\hline Amoxicillin-clavulanic acid & $111(72)$ & $50(56)$ & $61(94)$ & $<0.0001$ \\
\hline Trimethoprim sulfonamides & $130(84)$ & $67(74)$ & 63(97) & 0.0002 \\
\hline Nitrofurantoin & $9(6)$ & $6(7)$ & $3(5)$ & 0.5900 \\
\hline Fosfomycin & $15(10)$ & $8(9)$ & $7(11)$ & 0.6960 \\
\hline $\mathrm{fimH}$ & $155(100)$ & $90(100)$ & $65(100)$ & $>0.9999$ \\
\hline papA & $20(13)$ & $12(13)$ & $8(12)$ & 0.8509 \\
\hline papC & $75(48)$ & $42(47)$ & $33(51)$ & 0.6140 \\
\hline papEF & $37(24)$ & $18(20)$ & $19(29)$ & 0.1834 \\
\hline papGl & $6(4)$ & $3(3)$ & $3(5)$ & 0.6831 \\
\hline papGll & $70(45)$ & $33(37)$ & $37(57)$ & 0.0124 \\
\hline papGIII & $6(4)$ & $4(4)$ & $2(3)$ & 0.6632 \\
\hline$s f a / f o c$ & $20(13)$ & $11(12)$ & $9(14)$ & 0.7660 \\
\hline Afa & $15(10)$ & $7(8)$ & $8(12)$ & 0.3466 \\
\hline bmaE & $2(1)$ & $1(1)$ & $1(2)$ & 0.8246 \\
\hline fyuA & $37(24)$ & $20(22)$ & $17(26)$ & 0.5710 \\
\hline iutA & $86(55)$ & $45(50)$ & $41(63)$ & 0.1060 \\
\hline$f e o B$ & $76(49)$ & $40(44)$ & $36(55)$ & 0.1788 \\
\hline kpsmtll & $40(26)$ & $19(21)$ & $21(32)$ & 0.1160 \\
\hline kpsmt/ll & $4(3)$ & $2(2)$ & $2(3)$ & 0.4729 \\
\hline Usp & $22(14)$ & $10(9)$ & $12(18)$ & 0.1957 \\
\hline hlyA & $18(12)$ & 7 (8) & $11(17)$ & 0.0795 \\
\hline$C d t B$ & $11(7)$ & $6(7)$ & $5(8)$ & 0.8062 \\
\hline
\end{tabular}

Distribution of resistance and virulence traits among ESBL and non-ESBL producing uropathogenic $E$. coli $(N=155)$. The $p$ values were calculated by comparing different traits among ESBL producer's and non-ESBL producers

However, due to the emerging resistance to these antibiotics alternative therapeutic choices such as nitrofurantoin, fosfomycin and $\beta$-lactam inhibitors can be prescribed.

In this study, prevalence of ESBL genes was higher among ST131 and $90 \%$ of these strains were resistant to ceftazidime and cefotaxime. Likewise, resistance to ceftriaxone was confirmed in $77 \%$ of these strains. Because of their favorable safety, cephalosporins are considered important therapeutic choice for the treatment of uncomplicated UTIs among pregnant women [26].

Nitrofurantoin is a fluoroquinolone-sparing alternative antibiotic used for the treatment of uncomplicated cystitis
[27]. In recent years use of nitrofurantoin has increased steadily, particularly due to the resistance against trimethoprim/sulfamethoxazole and aminopencillins. Contraindication of ciprofloxacin in pregnancy and adverse impact on the gut flora favored the use of nitrofurantoin as an alternative treatment option for UTIs. In this study $13 \%$ of the ST-131 strains were resistant to nitrofurantoin.

We found that majority of the isolates belonging to the lineages ST405, ST168, ST29, ST69 and ST89 were multi-drug resistant. Percentage of MDR isolates was particularly higher among fluoroquinolonesresistant ST131 strains. Overall 59\% of the isolates 


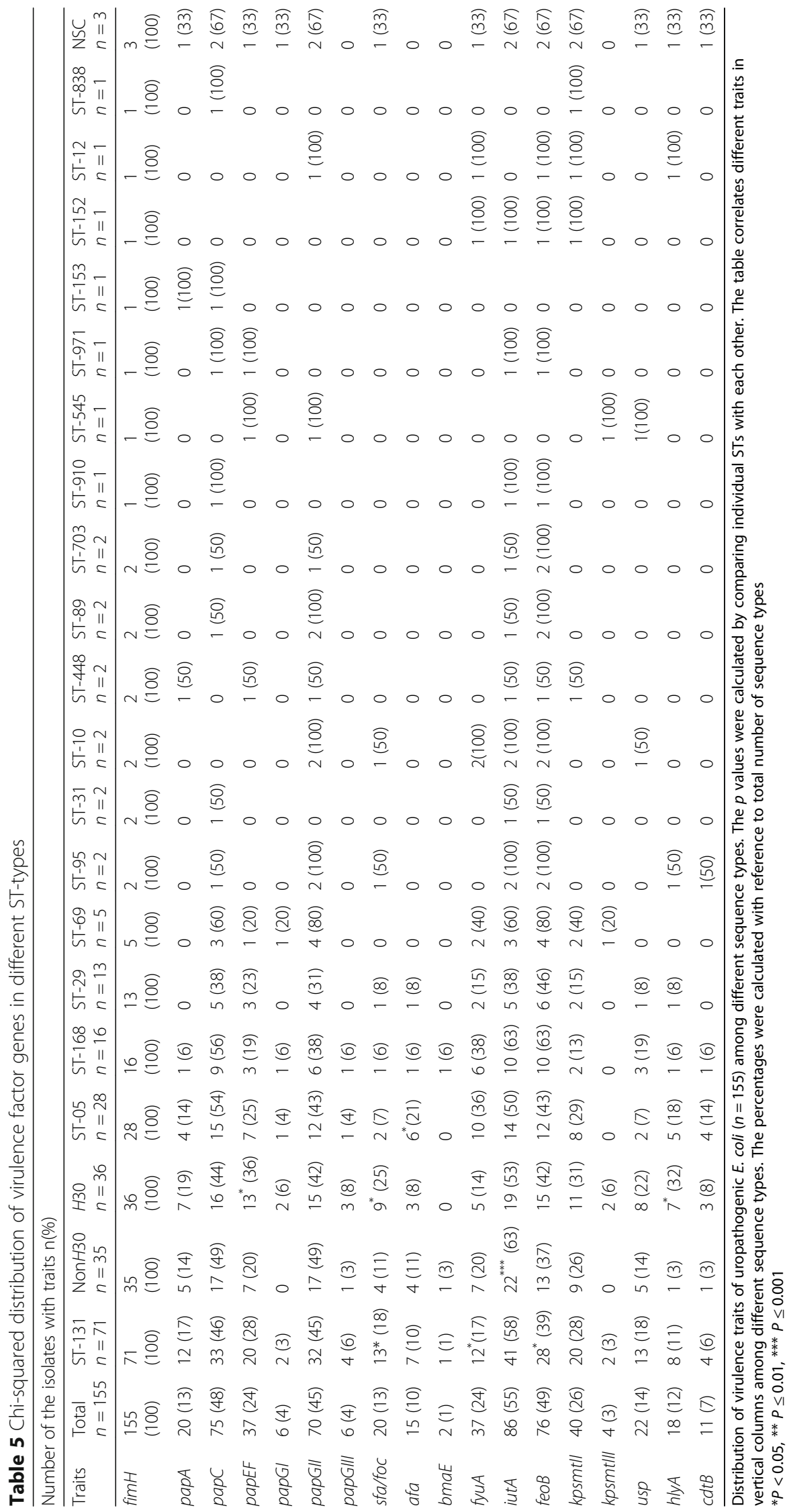


belonged to the phylogenetic group B2. A previous study from Pakistan confirmed that $50 \%$ of the UPEC isolates belonged to the phylogenetic group B2 [28]. Likewise, another study conducted in Pakistan reported that only $12 \%$ of the E. coli strains belonged to this phylogenetic group. These findings suggest that prevalence of phylogenetic group B2 may vary across different regions [29]. Few studies conducted previously in this region included phylogentic analysis of UPEC strains.

Phylogenetic group B2 strains are equipped with various VF genes relating to the extra-intestinal infections. These genes include P-fimbriae, S-fimbriae, haemolysin, aerobactin, $\mathrm{K} 1$ and $\mathrm{K} 5$ antigens and capsular antigen genes [30, 31]. A previous report focusing on the UPEC, in Pakistan described prevalence of various VF genes, including $h l y A, s f a D E$, papC,cnfl, eaeA and $a f a B C$ [29] While another study conducted on the rectal floral isolates of Pakistani children confirmed that virulence factors such as S-fimbriae, haemolysin, K-1 antigens and class III PapG adhesins were either very rare or completely absent [29]. In current study, UPEC strains of phylogenetic group B2, carried range of virulence factors, including genes for adhesins (fimH $100 \%$, papA $13 \%$, papC $47 \%$, papEF $21 \%$ pap GI $3 \%$, papGII $40 \%$, papGIII $4 \%$, sfalfoc $14 \%$, afa $11 \%$, bmaE 1\%), toxins (hlyA 7\%, cdtB 7\%) iron acquisition system (iutA 57\%, feoB43\%, fyuA 23\%) capsular proteins (kpsMTII 26\%, kpsMTIII 3\%) and uropathogenic specific protein (usp 14\%). We observed that the gene papGII was significantly associated with phylogenetic group B2 strains and association of papGII with pyelonephritis and bacteraemia in human has been confirmed earlier [32-34]. In the current study, fimbriae associated gene $f i m H$ was detected among $100 \%$ of the UPEC isolates Role of fimH in adhesion, invasion and formation of the intracellular bacterial communities (IBCs) has been described previously and its importance in the host pathogen interaction was confirmed by higher vulnerabilities of premenopausal women to UPEC infections [35]. In this study genes related to the adhesins (papEF, $s f a / f o c)$ and toxins ( $h l y A)$ were found to be strongly associated with ST131 H30 sub-clone. Recently hlyA in interaction with natural killer (NK) cells of urinary bladder was described [36]. Likewise, we witnessed significant association of the iron acquisition genes (ffyuA and $f e o B$ ) with ST131 lineage. The importance of gentic factors related to the iron acquisition system was shown by strong upregulation of these genes during UTIs [37]. Generally, E. coli strains causing UTI share similar properties in terms of phylogeny, sero-grouping and VF genes. However, other than genetic attributes of the virulence strains, host factors may play important role in the outcome of infection [38].

\section{Conclusion}

In conclusion it is the first report that highlights MDR ST131 as a predominant linage associated with UTI in Pakistan. ST131 and other scrutinized sequence types having MDR status among UTI isolates in Pakistan indicate considerable constraints on the empirical choice for the treatment of UTI. Alternative therapies and identification of effective prevention strategies-including antibiotic stewardship - are needed. As antibiotic resistance can be transferred from UPEC to other pathogens, more judicious use of antibiotics is required.

\section{Abbreviations}

ESBL: Extended spectrum beta lactamases; IBCs: Intracellular bacterial communities; MDR: Multidrug resistance; NK: Natural killer; ST: Sequence type; UPEC: Uropathogenic E. coli; UTI: Urinary tract infections; VF: Virulence factors

\section{Acknowledgements}

We thank Prof. Betsy Foxman, Hunein F. and Hilda Maassab Endowed Professor of Epidemiology Director, Center for Molecular and Clinical Epidemiology of Infectious Diseases at University of Michigan, USA for supporting this work.

\section{Authors' contributions}

DIJ and FB designed and supervised the study. Al and RZ conducted the bench work and assembled the data. TF helped in MIC determination. Al, GES, and ES helped in statistical and bioinformatics analysis. DIJ did analysis, interpretation and drafted the manuscript. All authors have read, contributed and approved the final manuscript.

\section{Funding}

Higher Education Commission Pakistan provided 6 month stipend and bench fee for the doctoral work of Ihsan Ali at Center for Molecular and Clinical Epidemiology of Infectious Diseases at University of Michigan, USA which enabled us to perform ST typing of strains (International Research Support Initiative Program IRSIP/2016). Work of Zara Rafaque was funded by HEC indigenous PhD scholarships covering monthly stipend University fee and lab reagents that helped us to screen VF and ESBL genes (HEC-21353961-2BM2-093). Higher Education Commission Pakistan played no direct role in collection, analysis, interpretation and publication of this work.

\section{Availability of data and materials}

The datasets used and/or analyzed during the current study available from the corresponding author on reasonable request.

\section{Ethics approval and consent to participate}

Ethical Review Board (ERB) of Pakistan Institute of Medical Sciences approved this study. Ethical Review Board approved verbal consent taken from all the patients.

\section{Consent for publication}

Not applicable.

\section{Competing interests}

The authors declare that they have no competing interests.

\section{Author details}

'Department of Microbiology, Faculty of Biological Sciences, Quaid-i-Azam University, Islamabad 45320, Pakistan. ${ }^{2}$ Alpha Genomics (Pvt) Ltd, Islamabad, Pakistan. ${ }^{3}$ Department of Biophysics, University of Michigan, Ann Arbor, MI 48109, USA. ${ }^{4}$ Department of Epidemiology, School of Public Health, University of Michigan, Ann Arbor, MI 48109, USA. ${ }^{5}$ Department of Medical Laboratory Technology (MLT), the University of Haripur, Abbottabad, Pakistan. 
Received: 12 April 2019 Accepted: 4 July 2019

Published online: 12 July 2019

\section{References}

1. Dehbanipour R, Rastaghi S, Sedighi M, Maleki N, Faghri J. High prevalence of multidrug-resistance uropathogenic Escherichia coli strains, Isfahan, Iran. J Nat Sci Biol Med. 2016;7(1):22.

2. Mittal $S$, Sharma $M$, Chaudhary U. Biofilm and multidrug resistance in uropathogenic Escherichia coli. Pathog Glob Health. 2015;109(1):26-9.

3. Tanvir R, Hafeez R, Hasnain S. Prevalence of multiple drug resistant Escherichia coli in patients of urinary tract infection registering at a diagnostic laboratory in Lahore Pakistan. Pak J Zool. 2012;44(3):707-12.

4. Johnson JR, Tchesnokova V, Johnston B, Clabots C, Roberts PL, Billig M, Riddell K, Rogers P, Qin X, Butler-Wu S. Abrupt emergence of a single dominant multidrug-resistant strain of Escherichia coli. J Infect Dis. 2013; 207(6):919-28.

5. Petty NK, Zakour NLB, Stanton-Cook M, Skippington E, Totsika M, Forde BM, Phan M-D, Moriel DG, Peters KM, Davies M. Global dissemination of a multidrug resistant Escherichia coli clone. Proc Natl Acad Sci U S A. 2014; 111(15):5694-9.

6. Shahzad N, Aslam B, Hussain I, Ijaz M, Rasool MH, Tasneem F, Hamid T, Tayyeb A, Hussain T. Distribution and Phylogenetic Analysis of Bacterial Isolates from Urinary Tract Infection Patients of Pakistan. Pak J Zool. 2016:48(6):1925-30.

7. Johnson JR, Johnston B, Clabots C, Kuskowski MA, Castanheira M. Escherichia coli sequence type ST131 as the major cause of serious multidrug-resistant E. coli infections in the United States. Clin Infect Dis. 2010;51(3):286-94.

8. Nicolas-Chanoine M-H, Bertrand X, Madec J-Y. Escherichia coli ST131, an intriguing clonal group. Clin Microbiol Rev. 2014;27(3):543-74.

9. Johnson JR, Johnston B, Thuras P, Launer B, Sokurenko EV, Miller LG. Escherichia coli Sequence Type 131 H30 Is the Main Driver of Emerging Extended-Spectrum- $\beta$-Lactamase-Producing E. coli at a Tertiary Care Center. mSphere. 2016;1(6):e00314-6.

10. Johnson JR, Menard M, Johnston B, Kuskowski MA, Nichol K, Zhanel GG. Epidemic clonal groups of Escherichia coli as a cause of antimicrobialresistant urinary tract infections in Canada, 2002 to 2004. Antimicrob Agents Chemother. 2009;53(7):2733-9.

11. Cagnacci S, Gualco L, Debbia E, Schito GC, Marchese A. European emergence of ciprofloxacin-resistant Escherichia coli clonal groups O25: H4ST 131 and 015: K52: H1 causing community-acquired uncomplicated cystitis. J Clin Microbiol. 2008;46(8):2605-12.

12. Hefzy EM, Hassuna NA. Fluoroquinolone-resistant sequence type 131 subgroups $\mathrm{O} 25 \mathrm{~b}$ and $\mathrm{O} 16$ among Extraintestinal Escherichia coli isolates from community-acquired urinary tract infections. Microb Drug Resist. 2017; 23(2):224-9

13. Clinical and Laboratory Standards Institute, 2014. Performance standards for antimicrobial susceptibility testing: 24th informational supplement. . Document M100-S24.

14. Clermont O, Bonacorsi S, Bingen E. Rapid and simple determination of theEscherichia coli phylogenetic group. Appl Environ Microbiol. 2000;66(10):4555-8.

15. Weissman SJ, Johnson JR, Tchesnokova V, Billig M, Dykhuizen D, Riddell K, Rogers P, Qin X, Butler-Wu S, Cookson BT. High-resolution two-locus clonal typing of extraintestinal pathogenic Escherichia coli. Appl Environ Microbiol. 2012;78(5):1353-60.

16. Tchesnokova V, Billig M, Chattopadhyay S, Linardopoulou E, Aprikian P, Roberts PL, Skrivankova V, Johnston B, Gileva A, Igusheva I. Predictive diagnostics for Escherichia coli infections based on the clonal association of antimicrobial resistance and clinical outcome. J Clin Microbiol. 2013;51(9):2991-9.

17. Clermont O, Dhanji H, Upton M, Gibreel T, Fox A, Boyd D, Mulvey MR, Nordmann P, Ruppé E, Sarthou JL. Rapid detection of the O25b-ST131 clone of Escherichia coli encompassing the CTX-M-15-producing strains. J Antimicrob Chemother. 2009;64(2):274-7.

18. del Castillo BR, Vinué L, Román E, Guerra B, Carattoli A, Torres C, MartínezMartínez L. Molecular characterization of multiresistant Escherichia coli producing or not extended-spectrum ß-lactamases. BMC Microbiol. 2013;13(1):84.

19. Bush KTP JG: CTX-M-type Beta-lactamases. Lahey Clinic. 2015.

20. Yun KW, Kim HY, Park HK, Kim W, Lim IS. Virulence factors of uropathogenic Escherichia coli of urinary tract infections and asymptomatic bacteriuria in children. J Microbiol Immunol Infect. 2014;47(6):455-61.

21. Alghoribi MF, Gibreel TM, Farnham G, Al Johani SM, Balkhy HH, Upton M. Antibiotic-resistant ST38, ST131 and ST405 strains are the leading uropathogenic Escherichia coli clones in Riyadh, Saudi Arabia. J Antimicrob Chemother. 2015;70(10):2757-62.

22. Peirano G, Pitout JD. Molecular epidemiology of Escherichia coli producing CTX-M $\beta$-lactamases: the worldwide emergence of clone ST131 O25: H4. Int J Antimicrob Agents. 2010;35(4):316-21.

23. Banerjee R, Robicsek A, Kuskowski MA, Porter S, Johnston BD, Sokurenko E, Tchesnokova V, Price LB, Johnson JR. Molecular epidemiology of Escherichia coli sequence type 131 and its $\mathrm{H} 30$ and H30-Rx subclones among extended-spectrum- $\beta$-lactamase-positive and-negative $\mathrm{E}$. coli clinical isolates from the Chicago region, 2007 to 2010. Antimicrob Agents Chemother. 2013;57(12):6385-8.

24. Lau SH, Reddy S, Cheesbrough J, Bolton FJ, Willshaw G, Cheasty T, Fox AJ, Upton M. Major uropathogenic Escherichia coli strain isolated in the northwest of England identified by multilocus sequence typing. J Clin Microbiol. 2008;46(3):1076-80.

25. Johnson JR, Clermont O, Johnston B, Clabots C, Tchesnokova V, Sokurenko E, Junka AF, Maczynska B, Denamur E. Rapid and specific detection, molecular epidemiology, and experimental virulence of the 016 subgroup within Escherichia coli sequence type 131. J Clin Microbiol. 2014;52(5):1358-65.

26. Nicolle L. Management of asymptomatic UTIs in women. Medscape Womens Health. 1996;1(3):4-4.

27. Hooton TM. Fluoroquinolones and resistance in the treatment of uncomplicated urinary tract infection. Int J Antimicrob Agents. 2003;22: $65-72$.

28. Bashir S, Haque A, Sarwar Y, Ali A, Anwar MI. Virulence profile of different phylogenetic groups of locally isolated community acquired uropathogenic $E$. coli from Faisalabad region of Pakistan. Ann Clin Microbiol Antimicrob. 2012;11(1):23.

29. Nowrouzian F, Östblom A, Wold A, Adlerberth I. Phylogenetic group B2 Escherichia coli strains from the bowel microbiota of Pakistani infants carry few virulence genes and lack the capacity for long-term persistence. Clin Microbiol Infect. 2009;15(5):466-72.

30. Abdi HA, Rashki A. Comparison of virulence factors distribution in uropathogenic E. coli isolates from phylogenetic groups B2 and D. Int J Enteric Pathog. 2014;2(4):1-5.

31. Er DK, Dundar D, Uzuner H, Osmani A. Relationship between phylogenetic groups, antibiotic resistance and patient characteristics in terms of adhesin genes in cystitis and pyelonephritis isolates of Escherichia coli. Microb Pathog. 2015;89:188-94.

32. Johnson JR. papG alleles among Escherichia coli strains causing urosepsis: associations with other bacterial characteristics and host compromise. Infect Immun. 1998;66(9):4568-71.

33. Johnson JR, Kuskowski MA, Gajewski A, Soto S, Horcajada JP, de Anta MTJ, Vila J. Extended virulence genotypes and phylogenetic background of Escherichia coli isolates from patients with cystitis, pyelonephritis, or prostatitis. The J Infect Dis. 2005;191(1):46-50.

34. Otto G, Sandberg T, Marklund B-I, Ulleryd P, Svanborg C. Virulence factors and pap genotype in Escherichia coli isolates from women with acute pyelonephritis, with or without bacteremia. Clin Infect Dis. 1993;17(3):448-56.

35. Foxman B, Brown P. Epidemiology of urinary tract infections: transmission and risk factors, incidence, and costs. Infect Dis Clin N Am. 2003;17(2):227-41.

36. Gur C, Coppenhagen-Glazer S, Rosenberg S, Yamin R, Enk J, Glasner A, BarOn Y, Fleissig O, Naor R, Abed J. Natural killer cell-mediated host defense against uropathogenic E. coli is counteracted by bacterial hemolysinAdependent killing of NK cells. Cell Host Microbe. 2013;14(6):664-74.

37. Hagan EC, Lloyd AL, Rasko DA, Faerber GJ, Mobley HL. Escherichia coli global gene expression in urine from women with urinary tract infection. PLoS Pathog. 2010;6(11):e1001187.

38. Takahashi A, Kanamaru S, Kurazono H, Kunishima Y, Tsukamoto T, Ogawa O, Yamamoto S. Escherichia coli isolates associated with uncomplicated and complicated cystitis and asymptomatic bacteriuria possess similar phylogenies, virulence genes, and O-serogroup profiles. J Clin Microbiol. 2006;44(12):4589-92.

\section{Publisher's Note}

Springer Nature remains neutral with regard to jurisdictional claims in published maps and institutional affiliations. 